\title{
Die klinische Diagnostik der Cannabisabhängigkeit bei Kindern und Jugendlichen - die jugendpsychiatrische Perspektive
}

\author{
Clinical Diagnosis of Cannabis Dependence in Children and \\ Adolescents - the Perspective of Child Psychiatrists
}

\section{Zusammenfassung}

Aufgaben der klinischen Kinder- und Jugendpsychiatrie sind die Früherkennung und Frühintervention bei psychischen Störungen, die Langzeitbehandlung chronifizierter Störungen, etwa aus dem schizophrenen Formenkreis, und die zielgerichtete psychiatrische Behandlung begleitender Störungen. Grundlage hierfür ist einerseits die Entwicklungspsychopathologie mit ihrem Krankheitsverständnis zwischen Vulnerabilität und Resilienz, andererseits eine standardisierte multiachsiale Diagnostik mit Integration psychiatrischer, somatischer und sozialer Befunde. Während CannabisKonsum für viele Jugendliche eine vorübergehende, überwiegend angenehm empfundene Erfahrung darstellt, finden sich in den Kinder- und jugendpsychiatrischen Kliniken Jugendliche, die erhebliche seelische Schäden mit Cannabis zu bekämpfen versuchen oder im Zusammenhang mit Cannabis-Konsum erlitten haben. Patienten, Eltern und Kostenträger erwarten zu Recht eine objektive und nachvollziehbare Diagnostik und leitlinienorientierte Therapiemethoden.

Schliisselwörter

Adoleszenz · Cannabis · Diagnostik

\section{Abstract}

The adolescent psychiatric approach to cannabis-related mental health problems generally concentrates on early detection of treatable psychiatric disorders and early out-patient intervention. As many chronic psychiatric disorders such as depression, ADHD, social phobia and psychosis coincide with cannabisabuse and dependency in adolescence, in-patient care may be needed, thus defining a special subgroup of severe comorbid patients. The multidimensional and multiaxial diagnostic criteria should be carefully applied to install a long-term multimodal treatment model in these severely disturbed patients, integrating psychiatric, psychosocial and substance related aspects. As chronic cannabis-related disorders seem to increase in adolescent psychiatric care, integrated treatment models based on careful diagnostics have to be developed.

Key words

Adolescence $\cdot$ Cannabis $\cdot$ diagnostics

\section{Einleitung}

Das psychische, körperliche und soziale Gefährdungspotenzial von intensivem Cannabis-Konsum ist in den letzten Jahren in den Vordergrund des öffentlichen Interesses gerückt. Es wurden ver- schiedene Konsummuster und Typologien entwickelt [1], die es im Public-Health-Bereich erleichtern, Interventionen zu planen.

In den Kliniken für Kinder- und Jugendpsychiatrie und Psychotherapie und hier speziell in den Versorgungskliniken zeigen

Institutsangaben

Kliniken für Kinder- und Jugendpsychiatrie und Psychotherapie, Vivantes-Humboldt-Klinikum,

Vivantes-Klinikum Hellersdorf

Korrespondenzadresse

Dr. med. Oliver Bilke · Kliniken für Kinder- und Jugendpsychiatrie und Psychotherapie,

Vivantes-Humboldt-Klinikum, Vivantes-Klinikum Hellersdorf · Frohnauerstr. 74-80 · 13467 Berlin .

E-mail: oliver.bilke@vivantes.de

Bibliografie

Suchttherapie 2005; 6: 102 - 107 @ Georg Thieme Verlag KG Stuttgart · New York

DOI 10.1055/s-2005-858617

ISSN 1439-9903 
sich parallel zu dieser Entwicklung immer häufiger auch jüngere Patienten ab elf Jahren mit manifester Cannabis-Abhängigkeit.

Während einzelne psychiatrische Auffälligkeiten bei intensivem Cannabis-Konsum (wie das so genannte amotivationale Syndrom) und auch die Diagnosemöglichkeiten einer Sucht bei unter 16-Jährigen noch kritisch diskutiert werden, ergibt sich praktisch die Notwendigkeit, die Betroffenen adäquat diagnostisch und therapeutisch zu unterstützen.

Eine sorgfältige multiaxiale Diagnostik [2] ist hierbei im Einzelfall unabdingbare Voraussetzung für eine langfristige Therapieplanung. Anspruchsvoll bleiben die unterschiedlichen Aspekte der individuellen Entwicklungspathologie, der Suchtmittelwirkung und der sozialen und familiären Rahmenbedingungen [3], die als komplexes Gefüge die Diagnose einer Abhängigkeit von Cannabis bestimmen.

Diagnostische Strategien bei Suchtkranken in der Kinder- und Jugendpsychiatrie

\section{Entwicklungspsychopathologie}

Die Entwicklungspsychopathologie kann mittlerweile als zentrale Grundlagenwissenschaft der Kinder- und Jugendpsychiatrie gelten $[4,5]$.

Sie betrachtet die Einflüsse der normalen Entwicklung auf die Genese und die Ausprägung psychopathologischer Symptome in unterschiedlichen Lebensaltern. Andererseits wird der Einfluss psychopathologischer Symptome auf den normalen Entwicklungsverlauf in den Fokus genommen. Ausgangspunkt der Entwicklungspsychopathologie ist eine interaktionistische Entwicklungstheorie, die davon ausgeht, dass ein aktives selbstmotiviertes und die Eigenentwicklung vorantreibendes Individuum mit einer ebenso aktiven fordernden und Einfluss nehmenden Umwelt in Interaktion steht (vgl. auch [6]).

Wesentliche Modellvorstellungen sind hierbei VulnerabilitätsStress-Modelle, wobei Vulnerabilität als erhöhtes Risiko zur Fehlentwicklung oder psychischen Dekompensation bei Anwesenheit von unabhängigen Risikobedingungen definiert wird. Der Vulnerabilität stehen Ressourcen im materiellen und psychosozialen Bereich gegenüber, wobei im Einzelfall die individuelle Resilienz, also die Fähigkeit mit widrigen Umgebungsfaktoren kreativ und positiv umzugehen, das klinische Bild bestimmt.

In diesem Gesamtkontext kann der Cannabis-Konsum als heutzutage in unserer Kultur notwendige Entwicklungsaufgabe gesehen werden, die von den meisten Jugendlichen bewältigt wird. Für vulnerable Jugendliche mit geringen Resilienzfaktoren ist der Cannabis-Konsum aber einerseits eine vorübergehende scheinbare Ressource gegenüber psychischen Erkrankungen, langfristig jedoch ein weiterer gravierender Risikofaktor.

Auf dem Boden der Entwicklungspsychopathologie sind insbesondere der Beginn des Suchtkonsums, die Suchtdynamik, die verwendeten Substanzen, aber auch die Interaktion mit anstehenden äußeren Aufgaben (Loslösung vom Elternhaus, schulische Integration etc.) von Bedeutung.

\section{Befunderhebung}

Die Basis jeglichen kinder- und jugendpsychiatrischen Handelns - auch und besonders im Suchtbereich - ist die multiaxiale Diagnostik nach dem multiaxialen Klassifikationsschema (MAS) [2].

Auf 6 Achsen werden systematisch die insbesondere für Kinder und Jugendliche relevanten Lebens- und Themenbereiche erarbeitet.

Das MAS umfasst die folgenden Achsen:

- Achse 1: klinisch-psychiatrisches Syndrom;

- Achse 2: umschriebene Entwicklungsstörungen;

- Achse 3: Intelligenzniveau;

- Achse 4: körperliche Symptomatik;

- Achse 5: aktuelle abnorme psychosoziale Umstände;

- Achse 6: Globalbeurteilung der psychosozialen Anpassung.

Bei einer Erkrankung im Zusammenhang mit Cannabis werden auf der Achse 1 die Kategorien F12.0 bis F12.9 verwendet (siehe Tab. 1).

Tab. 1 MAS/ICD-10-Klassifikation: Cannabis-induzierte psychische Störungen

\begin{tabular}{|l|l|}
\hline \multicolumn{1}{l}{ Störung } & Codierung \\
\hline akute Intoxikation & $\mathrm{F} 12.0$ \\
\hline schädlicher Gebrauch & $\mathrm{F} 12.1$ \\
\hline Abhängigkeitssyndrom & $\mathrm{F} 12.2$ \\
\hline Entzugssyndrom & $\mathrm{F} 12.3$ \\
\hline Entzug mit Delir & $\mathrm{F} 12.4$ \\
\hline psychotische Störung & $\mathrm{F} 12.5$ \\
\hline amnestisches Syndrom & $\mathrm{F} 12.6$ \\
\hline verzögertes psychotisches Syndrom & $\mathrm{F} 12.7$ \\
\hline Verhaltensstörungen & $\mathrm{F} 12.8$ \\
\hline nicht näher bezeichnete Störungen & $\mathrm{F} 12.9$ \\
\hline
\end{tabular}

Die diagnostischen Leitlinien der Cannabis-Abhängigkeit (F12.2) lauten im MAS:

\section{Abhängigkeitssyndrom}

Es handelt sich um eine Gruppe körperlicher, Verhaltens- und kognitiver Phänomene, bei denen der Konsum einer Substanz oder einer Substanzklasse für die betroffene Person Vorrang hat gegenüber anderen Verhaltensweisen, die von ihr früher höher bewertet wurden. Ein entscheidendes Charakteristikum der Abhängigkeit ist der oft starke, gelegentlich übermächtige Wunsch, Substanzen oder Medikamente (ärztlich verordnet oder nicht), Alkohol oder Tabak zu konsumieren.

\section{Diagnostische Leitlinien:}

Die Diagnose Abhängigkeit soll im Sinne der MAS nur gestellt werden, wenn mindestens einen Monat während des letzten Jahres drei oder mehr der folgenden Kriterien zusammen vorhanden waren oder sich wiederholt haben:

1. ein starker Wunsch oder eine Art Zwang, Substanzen oder Alkohol zu konsumieren; 
2. verminderte Kontrollfähigkeit bezüglich des Beginns, der Beendigung und der Menge des Substanz- oder Alkoholkonsums;

3. Substanzgebrauch mit dem Ziel, Entzugssymptome zu mildern und der entsprechenden positiven Erfahrung;

4. ein körperliches Entzugssyndrom;

5. Nachweis einer Toleranz; um die ursprünglich durch niedrigere Dosen erreichte Wirkung der Substanz hervorzurufen, sind zunehmend höhere Dosen erforderlich (eindeutige Beispiele hierfür sind die Tagesdosen von Alkoholikern und Opiatabhängigen, die Konsumenten ohne Toleranzentwicklung schwer beeinträchtigen würden oder sogar zum Tode führten);

6. ein eingeengtes Verhaltensmuster im Umgang mit Alkohol oder der Substanz wie z.B. die Tendenz, Alkohol an Werktagen wie an Wochenenden zu trinken und die Regeln eines gesellschaftlich üblichen Trinkverhaltens außer Acht zu lassen;

7. fortschreitende Vernachlässigung anderer Vergnügen oder Interessen zugunsten des Substanzkonsums;

8. anhaltender Substanz- oder Alkoholkonsum trotz Nachweises eindeutiger schädlicher Folgen; die schädlichen Folgen können körperlicher Art sein, wie z.B. Leberschädigung durch exzessives Trinken, oder sozial, wie Arbeitsplatzverlust durch eine substanzbedingte Leistungseinbuße, oder psychisch, wie bei depressiven Zuständen nach massivem Substanzkonsum.

An dieser Stelle sei aus klinischer Sicht bemerkt, dass die Kategorien zum großen Teil denen der erwachsenen Patienten ähneln bzw. direkt übernommen sind. Das in Deutschland eher für Forschungszwecke verwendete DSM-IV würdigt beispielsweise die Toleranzentwicklung und die körperlichen Entzugssymptome weniger und bezieht Letztere stärker auf den allgemeinen Lebenswandel der Patienten (veränderte Ess-, Bewegungs- und Schlafgewohnheiten etc.). Inwieweit hier ein steigender THC-Gehalt der genommenen Einzeldosen eine Rolle in der individuellen Symptomausprägung spielt, bleibt systematischen Untersuchungen vorbehalten.

Die Klassifikationsfrage ist klinisch als problematisch zu sehen, da die körperlichen und seelischen Besonderheiten gerade des jüngeren Jugendalters nicht genügend Berücksichtigung gefunden haben, z. B. was die teilweise sehr schnelle Entzugsdynamik und die gesteigerte Verstoffwechslung von Drogen bei Jugendlichen angeht.

Der adoleszente Organismus ist wesentlich schneller als der des Erwachsenen in der Lage, auch große und polytoxische Drogenmengen $\mathrm{zu}$ entgiften und danach eine scheinbare Symptomfreiheit zu zeigen. Auch sind die spezifischen Konsummuster Jugendlicher [1] eher dazu angetan, eine Abhängigkeit zu verschleiern. Der keinesfalls nur akademisch bedeutsame Übergang zwischen schädlichem Gebrauch und manifester Abhängigkeit bedarf in der Diagnostik der besonderen Aufmerksamkeit.

\section{Achse - psychiatrische Störungen}

Auf der 1. Achse MAS wird die psychiatrische Diagnose festgelegt. Hier gilt unter entwicklungspathologischem Aspekt der Leitsatz einer nicht zu frühen Festlegung auf langfristige Diagno- sen bei gleichzeitiger sachlicher Überprüfung, ob sich nicht bereits im Jugendalter eine schwerwiegende Persönlichkeitsstörung entwickelt, die ihrerseits die gesamte Prognose bestimmt.

Als häufige Begleitdiagnosen im Sinne der ICD-10 bei CannabisAbhängigkeit im Jugendalter sind zu nennen:

- Depressionen,

- soziale Phobien,

- Psychosen,

- Aufmerksamkeitsdefizitsyndrom,

- Essstörungen,

- Persönlichkeitsstörungen.

Für die Therapieplanung ist es wichtig, den prämorbiden Zustand des Patienten sorgfältig zu erfassen, was in den meisten Fällen durch Fremdanamnese von Familienmitgliedern oder auch Lehrern gelingt. Inwieweit eine psychiatrische Störung bereits vor einem manifesten Drogenkonsum vorlag und vielleicht eine sog. Selbstmedikation zu prüfen ist, oder ob der Konsum selbst psychische Störungen induziert hat, ist zu klären. Der klinische Alltag zeigt hier häufig schwer differenzierbare Mischbilder.

\section{Achse - Teilleistungsstörungen}

Eine Besonderheit der kinder- und jugendpsychiatrischen Diagnostik stellt die regelmäßige Einschätzung so genannter Teilleistungsstörungen (Lese-Rechtschreibschwäche, motorische Störungen, sensorische Störungen, Rechenschwäche) dar.

Diese Entwicklungsstörungen, die nur bei durchschnittlicher Intelligenz diagnostiziert werden können, beeinträchtigen das soziale Funktionieren je nach Ausprägungsgrad schwer, behindern schulische Weiterentwicklung oder Reintegration und führen unbehandelt im Einzelfall auch zu erheblichen sozialen Defiziten.

Insbesondere die Lese-Rechtschreibschwäche (Legasthenie/Dyslexie), die im Kindesalter prinzipiell gut beeinflussbar ist, hat für die Ausbildungsentwicklung des Jugendlichen größte Bedeutung.

Bedauerlicherweise ist festzustellen, dass sowohl in Suchteinrichtungen aber auch in Einrichtungen der Justiz der Anteil von Patienten mit Teilleistungsstörungen erheblich überrepräsentiert ist. Auch wenn die Verbindung zur Suchtentwicklung nicht i. S. einer Kausalität zu werten ist, sind die Risikofaktoren unbehandelter Teilleistungsstörungen nicht zu unterschätzen und es besteht ggf. auch im Jugendalter ein individueller Interventionsbedarf.

\section{Achse - Intelligenz}

Die Erhebung des Intelligenzbefundes durch einen differenziellen Intelligenztest kann die Weichen für sämtliche Therapieschritte stellen.

Scheinbar ist es dem Erfahrenen möglich, per Blickdiagnose und Einblick in die schulische Karriere einen Eindruck von der Intelligenz zu bekommen, dieser Eindruck ist aber meistens fehlerhaft und kann zu erheblichen Fehleinschätzungen in der Langzeitentwicklung führen.

Insbesondere diskrepante Intelligenzprofile - u.U. kombiniert mit Teilleistungsstörungen - ermöglichen es dem Klienten 
zwar in einzelnen Intelligenzbereichen (z.B. verbale Fähigkeiten) als durchschnittlich oder sogar überdurchschnittlich $\mathrm{zu}$ wirken, in anderen wichtigen Bereichen, die die Umsetzung von Therapie und Hilfsmaßnahmen angehen (planerische Intelligenz) versagt der Patient dann aber scheinbar unerklärlich.

Vielerlei gescheiterte Therapieansätze und psychosoziale Interventionen sind darauf zurückzuführen, dass ein zu anspruchsvolles oder zu einfaches intellektuelles Niveau der Kommunikation mit dem Patienten gewählt wurde.

Hinzu kommt die Tatsache, dass durch depressive Störungen, Psychosen, hyperkinetische Störungen, aber auch durch den Suchtmittelkonsum selbst verursachte neuropsychologische Auffälligkeiten die praktisch einsetzbare Intelligenz des Klienten stark beeinträchtigt ist. Erst nach einer Entgiftung bzw. längerfristigen Entwöhnungsphase kann dann ein vernünftiges Urteil über das Intelligenzniveau gegeben werden.

\section{Achse - körperliche Erkrankungen}

Auf der 4. Achse MAS (somatische Störung) sind sämtliche Auffälligkeiten im Entzugserleben bzw. in der Craving-Phase, aber auch langfristige körperliche Erkrankungen des Patienten wie die häufige chronische Bronchitis zu erfassen.

Während vor einigen Jahren noch ein Cannabis-Entzug als Faktum selbst diskutiert und bezweifelt wurde, zeigt sich aus der Optik der Versorgungskliniken ein zwar nicht spezifisches, aber eindeutiges Bild des akuten Cannabis-Entzugs [7].

Folgende Symptome tauchen - anderen Entzugssymptomatiken nicht unähnlich - auf:

- Schlafstörungen,

- bizarre Träume,

- Zittrigkeit,

- innere Unruhe,

- Anspannung,

- Schwitzen,

- allg. Gefühl des Unwohlseins,

- Erkältungsneigung,

- Spannungsgefühle am Körper.

Qualitative und quantitative Drogentests und in begrenztem Maße auch Schnelltests runden das diagnostische Bild ab.

Einzeln sind sie aber nicht in der Lage, Auskunft über das Vorliegen einer Abhängigkeit oder einer schweren Suchtentwicklung zu liefern, da immer noch Messfehler die Befunde beeinträchtigen, die andererseits aber auch oft Abbild der aktuellen Suchtdynamik bzw. des aktuellen Suchtmittelkonsums sind und nicht langfristige Tendenzen abbilden können.

Die Haaranalyse kann dies zwar gerade auch unter forensischem Aspekt, sagt aber ihrerseits nichts aus über die wichtigen Faktoren wie Toleranzentwicklung, Dosissteigerung oder zwanghaften Gebrauch.

\section{Achse - psychosoziale Faktoren}

Auf der 5. Achse MAS (abnorme psychosoziale Umstände) werden sämtliche Risikofaktoren, die eine kindliche und jugendliche
Entwicklung beeinflussen können, aufgelistet, um i. S. eines komplexen Risikofaktorenmodells die individuelle Belastung des Patienten abbilden zu können (vgl. hierzu umfassend [3]).

Bei Suchtpatienten werden besonders häufig die folgenden abnormen psychosozialen Umstände gefunden:

- abweichende Elternsituation (Trennung, Scheidung, Patchwork-Familie),

- Tod eines Elternteils,

- psychiatrische Erkrankungen eines Elternteils,

- körperliche Erkrankungen eines Elternteils,

- institutionelle Erziehung,

- sexueller Missbrauch,

- Migration,

- verzerrte intrafamiliäre Kommunikation.

Alle diese Einzelfaktoren sind in der Lage, aus sich heraus eine erhebliche Belastung der Entwicklung darzustellen. Insbesondere der sexuelle Missbrauch, gerade der sequenzielle intrafamiliäre andauernde Missbrauch, führt häufig zu weiteren psychischen Störungen wie einer posttraumatischen Belastungsstörung, die ihrerseits ein Suchtgeschehen mitbeeinflussen kann [8].

Insgesamt interagieren besonders die Probleme auf der ersten und der fünften Achse des MAS miteinander.

\section{Achse - Schweregrad}

Mit dem Begriff des allgemeinen Schweregrades der Störung insgesamt auf der 6 . Achse soll eingeschätzt werden, wie stark und in welchen Entwicklungsfeldern bzw. bei welchen Entwicklungsaufgaben eine besondere Beeinträchtigung des Patienten auffällt.

In dieser Kategorie werden die Symptomausprägung und vor allem der Einfluss auf verschiedene Lebensbereiche der Symptomatik erfasst.

Es geht um die Einschätzung der Beeinträchtigung in den Bereichen Schule, Gleichaltrige, Familie, im allgemeinen Leistungsbereich und in anderen für das Kind altersrelevanten psychosozialen Aufgaben.

Diese allgemeine Schweregradeinschätzung („global assessment of functioning“) ist besonders bei chronifizierten psychischen Störungen von Bedeutung, da auch bei gut gelingender Therapie u.U. die Erkrankung selbst nicht vollständig behandelt werden kann, sehr wohl aber der Schweregrad und damit die subjektive Lebensqualität für den Patienten erheblich beeinflusst werden kann.

Dies trifft insbesondere für Persönlichkeitsstörungen und manisch-depressive Erkrankungen sowie Psychosen aus dem schizophrenen Formenkreis zu.

\section{Eingesetzte Instrumente}

Für sämtliche psychiatrischen Diagnosen, für die allgemeine Erhebung des Befundes, aber insbesondere für die Erfassung der Teilleistungsstörung, der Intelligenzstörungen, der körperlichen Störungen und auch einzelner psychosozialer Merkmale 
liegen umfangreiche Manuale und testpsychologische Untersuchungsmethoden vor (vgl. [9]).

Basis jeglicher Befunderhebung ist die sorgfältige persönliche Anamnese und Exploration und insbesondere bei suchterkrankten Jugendlichen das kritische Hinterfragen der Angaben des Patienten und seiner Umwelt.

Während einige Autoren darauf hinweisen, dass über computergestützte standardisierte Fragebogenverfahren vor allem das Suchtverhalten und der konkrete Drogenkonsum gut zu erfassen sind, zeigen sich die individuelle Suchtdynamik, die für die Therapieplanung wichtige Entwicklung der Abhängigkeit sowie alle schambesetzten Themen (sexueller Missbrauch, Vernachlässigung etc.) nur im persönlichen mehrfachen Gespräch. Häufig tauchen wichtige anamnestische Angaben i. S. einer Prozessdiagnose erst nach mehreren Wochen der gemeinsamen Kooperation auf.

Ideal ist also die Kombination von individueller Anamneseerhebung, ggf. Einsatz standardisierter Untersuchungsinstrumente für den Suchtbereich (z.B. CIDI-DIAX) und für die jeweilige psychiatrische Fragestellung des einzelnen Patienten und seine Störung zugeschnittenen Fragebogeninstrumenten, wie z.B. Depressions- oder Angstfragebögen wie DIKJ oder AFS.

Im Forschungssetting sind selbstverständlich andere Instrumente einsetzbar, für den klinischen Gebrauch empfehlen sich einige wenige robuste und veränderungssensitive Instrumente, da bei kurzer Behandlungsdauer auftretende Effekte schnell erfasst werden müssen. Als klinisch besonders hilfreich haben sich erwiesen:

- ASI (addiction severity index),

- YSR (youth self report),

- DIKJ (Depressionsinventar für Kinder und Jugendliche),

- AFS (Angstfragebogen für Schüler),

- DISYPS (diagnostisches System für psychische Störungen im Kindes- und Jugendalter),

- Conners-Skalen (Erfassung von Hyperaktivität).

\section{Komorbidität und Therapieansätze}

Im Kindes- und Jugendalter sind Suchtstörungen ohne jegliche begleitende psychiatrische Erkrankung oder auch zugrunde liegende psychiatrische Erkrankung in den Versorgungskliniken nur in wenigen Fällen zu finden.

Dies zeigt sich auf den Spezialstationen, aber auch in der regulären Ambulanz und bei der Nachuntersuchung von Sucht- und Jugendhilfeklienten.

Während bei den höchst seltenen direkt Cannabis-induzierten psychotischen Zuständen prämorbid offenbar völlig unauffälliger Jugendlicher die Wirkung der Substanz ganz im Vordergrund der Ätiopathogenese steht, findet sich bei der deutlichen Mehrzahl der stationären Patienten ein teils jahrelanges komplexes Wechselspiel aus schleichend beginnenden psychiatrischen Störungen, erstem Cannabis-Konsum als (unbewusster) Copingstrategie, sekundärer Dosissteigerung und Abhängigkeitsent- wicklung und dann substanzinduzierten Auffälligkeiten. Eine entwicklungspsychopathologische Modellbildung, die zeitliche, suchtdynamische und entwicklungspsychologische Faktoren integriert, steht hier noch aus.

Der Diagnostik der komorbiden Störungen kommt daher für die Gesamtbehandlungsplanung zentrale Bedeutung zu, denn nach der in den meisten Fällen notwendigen Entgiftungsphase treten diese Störungen in den Vordergrund, beeinflussen die Therapie, das Cravingverhalten und insgesamt die Einsichtsfähigkeit des Patienten in die weiteren notwendigen Schritte (vgl. [10]).

Die wichtigsten Störungen im klinischen Alltag sind Depressionen, das Aufmerksamkeitsdefizitsyndrom (ADS; [11]) und soziale Ängste.

Eine ausreichend dosierte und kontinuierliche pharmakologische Behandlung des ADS mit Methylphenidat (z. B. Ritalin oder Medikinet) oder neuerdings mit Atomoxetin (Strattera) kann die Gesamtprognose erheblich verbessern.

Besorgniserregend ist insbesondere bei schizophrenen Patienten die Tatsache, dass sich in den klinischen Populationen kaum noch jugendliche Patienten ohne einen Cannabis-Abusus bzw. eine Cannabis-Abhängigkeit finden lassen.

Besonderes Augenmerk ist auch auf Jugendliche mit Störungen des Sozialverhaltens [12] und begleitenden psychiatrischen Störungen wie Depression zu richten, da diese aufgrund ihrer mangelnden Impulssteuerungsfähigkeit und niedrigen Frustrationsschwelle besonders zu sedierenden Substanzen wie Alkohol oder Cannabis neigen, um auf diesem Wege bewusst oder unbewusst ihre Impulsschwankungen zu steuern. Entgiftungen unter Zwangsszenarien können hier zu teilweise dramatischen Entäußerungen der gestauten Aggressivität führen.

Auch hier sind mit selektiven Serotonin-re-uptake-Hemmern wie Fluoxetin (Fluctin) und vor allem mit Risperidon (Risperdal) in niedriger Dosierung medikamentöse Erfolge zu erzielen.

Insgesamt hängt der nachhaltige Nutzen für den Patienten von der schrittweise geplanten und kontinuierlichen Therapiegestaltung auf dem Boden einer sorgfältigen multiaxialen Diagnostik ab.

Individuelle Konsummuster, die Entwicklung jugendlicher Risikoverhaltensweisen im Allgemeinen, gesellschaftliche Trends und die Parameter der Substanz Cannabis selbst bestimmen in einem dynamischen Wechselspiel den Kontext für das Individuum und seine Entwicklungsmöglichkeiten [13].

Hier schließt sich der Kreis einer interdisziplinären Herangehensweise an diese Problematik.

\section{Literatur}

${ }^{1}$ Soellner R, Kleiber D. Cannabisgebrauch in Deutschland - Konsumausmaß und Konsummuster. In: akzept e.V. (Hrsg). Gesellschaft mit Drogen - Akzeptanz im Wandel. Berlin: Verlag für Wissenschaft und Bildung, 2001: 147-161 
${ }^{2}$ Remschmidt H, Schmidt MH. Multiaxiales Klassifikationsschema für psychische Störungen des Kindes- und Jugendalters nach ICD-10 der WHO. Bern: Huber, 1994

${ }^{3}$ Thomasius R, Küstner U. Familie und Sucht Stuttgart: Schattauer, 2005: $27-38$

${ }^{4}$ Resch F. Entwicklungspsychopathologie. Weinheim: Beltz, 1999

${ }^{5}$ Herpertz-Dahlmann G, Resch F, Warnke A et al. Entwicklungspsychiatrie. Stuttgart: Schattauer, 2004

${ }^{6}$ Resch F, Fegert J, Buchmann J. Grundzüge der Diagnostik. In: Eggers C, Fegert F, Resch F (Hrsg). Psychiatrie und Psychotherapie des Kindesund Jugendalters. Heidelberg: Springer, 2003: 109-144

${ }^{7}$ Schneider U. Cannabisabhängigkeit bei Jugendlichen und jungen Erwachsenen. In: Möller C (Hrsg). Drogenmissbrauch im Jugendalter. Göttingen: Vandenhoeck und Ruprecht, 2005: 63-81

${ }^{8}$ Richter-Appelt H, Moldzio A. Sexualität und sexueller Missbrauch. In: Thomasius R, Küstner U (Hrsg). Familie und Sucht. Stuttgart: Schattauer, 2005: 27 - 38

${ }^{9}$ Deutsche Gesellschaft für Kinder- und Jugendpsychiatrie und Psychotherapie, Bundesarbeitsgemeinschaft leitender Klinikärzte für Kinder- und Jugendpsychiatrie und Psychotherapie, Berufsverband der Ärzte für Kinder- und Jugendpsychiatrie und Psychotherapie. Leitlinien zu Diagnostik und Therapie von psychischen Störungen im Säuglings-, Kindes- und Jugendalter. Köln: Dt. Ärzte-Verlag, 2004

${ }^{10}$ Möller C. Stationäre und ambulante Therapieangebote für drogenabhängige Jugendliche. In: Möller C (Hrsg). Drogenmissbrauch im Jugendalter. Göttingen: Vandenhoeck und Ruprecht, 2005: 63-81

${ }^{11}$ Biederman J, Wilens T, Mick E et al. Is ADHD a risk factor for psychoactive substance use disorders? Findings from a four-year prospective follow-up study. J Amer Acad Child Adolesc Psychiatry 1997; 36: $21-29$

12 Thompson LL, Riggs PD, Mikulich SK et al. Contribution of ADHD symptoms to substance problems and delinquency in conduct-disordered adolescents. J Abnorm Child Psychology 1996; 24: 325 - 347

${ }^{13}$ Kleiber D, Soellner R. Cannabiskonsum: Entwicklungstendenzen, Konsummuster und Risiken. Weinheim: Juventa, 1998 